\title{
THREE-MANIFOLDS WITH FUNDAMENTAL GROUP A FREE PRODUCT
}

\author{
BY WILLIAM JACO
}

Communicated by William Browder, April 7, 1969

1. Introduction. The purpose of this paper is to announce some results concerning the structure of a compact 3-manifold $M$ (possibly with boundary) where $\pi_{1}(M)$ is a free product. Related questions for $M$ closed have been considered in [1], [2], [4], [6], [8].

We use the term map to mean continuous function. If $M$ is a manifold, we use $\mathrm{Bd} M$ and Int $M$ to stand for the boundary and interior of $M$, respectively. The disk $D$ is said to be properly embedded in the 3-manifold $M$ if

$$
D \cap \operatorname{Bd} M=\operatorname{Bd} D .
$$

The compact 3-manifold $H_{n}$ is called a handlebody of genus $n$ if $H_{n}$ is the regular neighborhood of a finite connected graph having Euler characteristic $1-n$.

The combinatorial terminology is consistent with that of [9]. The terms in group theory may be found in [3]. Furthermore, all maps and spaces are assumed to be in the PL category.

\section{Bounded Kneser Conjecture.}

THEOREM 2.1. Let $M$ denote a compact 3-manifold with nonvoid boundary where $\pi_{1}(M) \approx A * B$, a free product. Then there is a compact 3-manifold $M^{\prime}$ with nonvoid boundary so that

(i) $M^{\prime}$ has the same homotopy type as $M$, and

(ii) there is a disk $D^{\prime}$ properly embedded in $M^{\prime}$ where $M^{\prime}-D^{\prime}$ consists of two components $M_{1}$ and $M_{2}$ with $\pi_{1}\left(M_{1}\right) \approx A$ and $\pi_{1}\left(M_{2}\right) \approx B$.

OUTLINE OF PROOF. Let $K_{A}$ and $K_{B}$ denote CW-complexes with $\pi_{1}\left(K_{G}\right) \approx G$ and $\pi_{n}\left(K_{G}\right)=0, n \geqq 2, G=A, B$. Let $p$ denote a point not in $K_{A} \cup K_{B}$. Define $\bar{K}_{A}$ and $\bar{K}_{B}$ as the mapping cylinders of maps from $p$ into $K_{A}$ and $K_{B}$, respectively. Let $K$ denote the CW-complex obtained by identifying the copy of $p$ in $\bar{K}_{A}$ with the copy of $p$ in $\bar{K}_{B}$. It follows that $\pi_{1}(K) \approx A * B$ and $\pi_{n}(K)=0, n \geqq 2$ (see [1, p. 669]).

There is a simplicial map $f$ of $M$ into $K$ ( $K$ may be chosen so that any finite collection of cells in $K$ has a simplicial subdivision) so that $f_{*}$ is an isomorphism of $\pi_{1}(M)$ onto $\pi_{1}(K)$.

Lemma A. Let $M, K, f, p$ be as above. There is a map $g: M \rightarrow K$ so that

(i) $g$ is homotopic to $f$ relative to a base point of $\pi_{1}(M)$, and 
(ii) each component of $g^{-1}(p)$ is a disk properly embedded in $M$.

If $g^{-1}(p)$ has only one component, then we are finished. Otherwise, we continue in the following way.

For $M, K$, and $p$ as above, the map $g: M \rightarrow K$ is said to be reduced if each component of $g^{-1}(p)$ is a disk properly embedded in $M$. If $g$ is reduced, the complexity of $g,{ }^{*}(g)$, is the number of components of $g^{-1}(p)$. Using this notation we wish to find a compact 3-manifold $M^{\prime}$ having the same homotopy type as $M$ and a reduced map $g^{\prime}: M^{\prime} \rightarrow K$ with ${ }^{*}\left(g^{\prime}\right)=1$ and $g_{*}^{\prime}$ an isomorphism.

We are able to find a handlebody, $H_{n}$, of genus $n$ in $M$ and 3-cells $B_{1}, \cdots, B_{k}$ so that for each $i=1, \cdots, k$,

$$
B_{i} \cap H_{n} \subset \operatorname{Bd} B_{i} \cap \operatorname{Bd} H_{n}=A_{i}
$$

is an annulus, $B_{i} \cap g^{-1}(p)=\varnothing, B_{i} \cap B_{j}=\varnothing, i \neq j$, and $M=H_{n} \cup B_{1}$ $\cup \ldots \cup B_{k}$. Under these conditions we say $\left(H_{n}, B_{1}, \cdots, B_{k}\right)$ is a Heegaard splitting for $M$ relative to $g$.

A path $\gamma$ in $\mathrm{Bd} H_{n}$ is called a binding tie (see [7]) if $\gamma \cap g^{-1}(p)$ consists of the endpoints of $\gamma$, the endpoints of $\gamma$ are in distinct components of $g^{-1}(p)$, and the loop $g(\gamma)$ based at $p$ is contractible in $K$ (actually, since $g(\gamma) \subset \bar{K}_{1}$ or $\bar{K}_{2}, g(\gamma)$ is contractible in $\bar{K}_{1}$ or $\bar{K}_{2}$ ).

LEMmA B. If $M, g, p$ are as in Lemma A and $\#(g)>1$, then there is a Heegaard splitting $\left(H_{n}, B_{1}, \cdots, B_{k}\right)$ of $M$ relative to $g$ and a nonsingular (i.e. an arc) binding tie in $\mathrm{Bd} H_{n}$.

We are now able to obtain a compact 3-manifold $M^{\prime}$ having the same homotopy type as $M$ and a map

$$
g^{\prime}: M^{\prime} \rightarrow K
$$

so that

(i) $g_{*}^{\prime}$ is an isomorphism of $\pi_{1}\left(M^{\prime}\right)$ onto $\pi_{1}(K)$,

(ii) $g^{\prime}$ is reduced,

(iii) ${ }^{*}\left(g^{\prime}\right)=\#(g)$, and

(iv) there is a Heegaard splitting

$\left(H_{n}^{\prime}, B_{1}^{\prime}, \cdots, B_{k}^{\prime}\right)$ of $M^{\prime}$ relative to $g^{\prime}$ and a nonsingular binding tie $\gamma^{\prime}$ in $\operatorname{Bd} H_{n}^{\prime}$ so that $\gamma^{\prime} \cap B_{i}^{\prime}=\varnothing$ for each $i=1, \cdots, k$.

LEMмa C. If $M^{\prime}, b^{\prime}, \gamma^{\prime}, p$ are as above, then there is a map $h^{\prime}: M^{\prime} \rightarrow K$ so that

(i) $h^{\prime}$ is homotopic to $g^{\prime}$ relative to a base point for $\pi_{1}\left(M^{\prime}\right)$,

(ii) $h^{\prime}$ is reduced, and

(iii) ${ }^{*}\left(h^{\prime}\right)<{ }^{*}\left(g^{\prime}\right)$. 
The following example shows that we cannot replace "homotopy type" by "homeomorphic" in part (i) of the previous theorem.

Let

$$
N=S^{1} \times S^{1} \times I
$$

where $S^{1}$ is the 1 -sphere and $I$ the unit interval. Let

$$
T_{0}=S^{1} \times S^{1} \times\{0\} \text { and } T_{1}=S^{1} \times S^{1} \times\{1\} .
$$

Choose disks $D_{0} \subset T_{0}$ and $D_{1} \subset T_{1}$. Let $B$ denote the unit 3-cell. Choose disks $D_{0}^{\prime}$ and $D_{1}^{\prime}$ in $\operatorname{Bd} B$ so that $D_{0}^{\prime} \cap D_{1}^{\prime}=\varnothing$. Let $h$ denote an orientation reversing homeomorphism from the pair $\left(D_{0}^{\prime}, D_{1}^{\prime}\right)$ to $\left(D_{0}, D_{1}\right)$. Define

$$
M=N \underset{h}{\cup} B,
$$

the 3-manifold obtained from the disjoint union of $N$ and $B$ by identifying $x$ with $h(x), x \in D_{0}^{\prime} \cup D_{1}^{\prime}$.

It is an elementary exercise using Van Kampen's Theorem to see that

$$
\pi_{1}(M) \approx(Z \times Z) * Z
$$

where $Z$ is the infinite cyclic group. The 3-manifold $M$ is irreducible; i.e. $M$ is irreducible if each polyhedral two-sphere in $M$ bounds a 3 -cell in $M$. Using this fact we obtain

Proposition 2.2. If $M$ is the 3-manifold constructed above and $D$ is a disk properly embedded in $M$ so that $M-D$ consists of two components $M_{1}$ and $M_{2}$, then the closure of either $M_{1}$ or $M_{2}$ is a 3-cell.

COROLlaRY 2.3. If $M$ is a compact 3-manifold and

$$
\pi_{1}(M) \approx A * B,
$$

then there are compact 3-manifolds $M_{1}$ and $M_{2}$ so that $\pi_{1}\left(M_{1}\right) \approx A$ and $\pi_{1}\left(M_{2}\right) \approx B$.

\section{Splitting along a disk.}

THEOREM 3.1. Let $M$ denote a compact 3-manifold with nonvoid, connected boundary. Suppose that each disk D properly embedded in $M$ separates $M$. If

$$
\pi_{1}(M) \approx A * B,
$$

then there is a disk $D$ properly embedded in $M$ so that $M-D$ consists of two components $M_{1}$ and $M_{2}$ with $\pi_{1}\left(M_{1}\right) \approx A$ and $\pi_{1}\left(M_{2}\right) \approx B$. 
OUTLINE OF PROOF. We proceed much along the same lines as in Theorem 2.1 through Lemma B of that theorem. Hence, borrowing from the notation of Theorem 2.1, suppose $g: M \rightarrow K$ is a map so that $g_{*}$ is an isomorphism and $g$ is reduced. We assume $g^{-1}(p)$ has more than one component (if $g^{-1}(p)$ has only one component, then we are finished).

Let $\left(H_{n}, B_{1}, \cdots, B_{k}\right)$ be a Heegaard splitting for $M$ relative to $g$. Suppose $\gamma$ is a nonsingular binding-tie in $\mathrm{Bd} H_{n}$.

LEMMA D. Using the above notation, there is a map $h: M \rightarrow K$ so that

(i) $h$ is homotopic to $g$ relative to a base point for $\pi_{1}(M)$,

(ii) $h$ is reduced, and

(iii) $\#(h)<\#(g)$.

THEOREM 3.2. Let $M$ denote a compact 3-manifold with nonvoid, connected boundary. If $\pi_{1}(M) \approx Z * G$, then there is a disk $D$ properly embedded in $M$ so that $M-D$ is connected and $\pi_{1}(M-D) \approx G$.

Proof. It is sufficient to show that there is a disk $D$ properly embedded in $M$ so that $M-D$ is connected (see the remarks on p. 27, vol. II of [3]).

If each disk properly embedded in $M$ separates $M$, then by Theorem 3.1 there is a disk $D$ properly embedded in $M$ so that $M-D$ has two components $M_{1}$ and $M_{2}$ with $\pi_{1}\left(M_{1}\right) \approx Z$ and $\pi_{1}\left(M_{2}\right) \approx G$. However, we have

LEMMA E. If $M_{1}$ is a compact 3-manifold with nonvoid boundary and $\pi_{1}\left(M_{1}\right) \approx Z$, then there is a disk $D$ properly embedded in $M_{1}$ so that $M_{1}-D$ is connected.

We conclude in any case that the desired disk $D$ may be obtained in $M$.

A group $G$ is said to be freely reduced if whenever $G \approx G_{1} * G_{2}$ then neither $G_{1}$ nor $G_{2}$ is a free group.

THEOREM 3.3. Let $M$ denote a compact 3-manifold with nonvoid, connected boundary. Suppose $\pi_{1}(M) \approx A * B$, a free product, and $\pi_{1}(M)$ is freely reduced. Then there is a disk $D$ properly embedded in $M$ so that $M-D$ consists of two components $M_{1}$ and $M_{2}$ with $\pi_{1}\left(M_{1}\right) \approx A$ and $\pi_{1}\left(M_{2}\right) \approx B$.

4. Another proof of Kneser's Conjecture. For an account of Kneser's Conjecture see [5] especially $\$ \$ 3,12,15,17$, and 20. Also, see [1], [2], [6]. Whitehead [8] was the first to obtain a satisfactory proof of Kneser's Conjecture. 
The group $G$ is said to be indecomposable if whenever $G \approx G_{1} * G_{2}$, then either $G_{1}$ or $G_{2}$ is the trivial group. If

$$
G \approx A_{1} * \cdots * A_{n}
$$

where each $A_{i}$ is indecomposable we call $A_{1} * \ldots * A_{n}$ a decomposition of $G$. Each finitely generated group has a decomposition which is unique up to order and isomorphism [3].

Kneser Conjecture. Let $M$ denote a closed 3-manifold. Then

$$
\pi_{1}(M) \approx A * B,
$$

a free product, iff there is a polyhedral 2-sphere $S$ in $M$ so that $M-S$ consists of two components $M_{1}$ and $M_{2}$ with $\pi_{1}\left(M_{1}\right) \approx A$ and $\pi_{1}\left(M_{2}\right) \approx B$.

We obtain Kneser's Conjecture as a corollary to

Theorem 4.1. Suppose $M$ is a closed 3-manifold and

$$
\pi_{1}(M) \approx A_{0} * A_{1} * \cdots * A_{k}
$$

is a decomposition of $\pi_{1}(M)$. Then there is a mutually exclusive collection of polyhedral 2-spheres $S_{1}, \cdots, S_{k}$ in $M$ so that $M-U_{1}^{k} S_{i}$ consists of $k+1$ components $N_{0}, \cdots, N_{k}$ with $\pi_{1}\left(N_{i}\right) \approx A_{i}$ for $0 \leqq i \leqq k$.

PROof. We proceed by induction on the integer $k$ where $k \geqq 1$. The general situation is no more difficult than the situation $k=1$.

Let $C$ denote a 3 -cell in $M$. Let $M^{\prime}=M$-Int $C$. Then $\pi_{1}\left(M^{\prime}\right)$ $\approx \pi_{1}(M)$. There are two cases to consider.

Case $1 . \pi_{1}(M)$ is not freely reduced.

We choose the notation in this case so that $A_{0} \approx Z$, the infinite cyclic group. Applying Theorem 3.2 we find a disk $D$ properly embedded in $M^{\prime}$ so that $M^{\prime}-D$ is connected. Hence, we are able to find a 2-sphere $S$ in $M$ so that $D \subset S$ and $M-S$ is connected. We have

Lemma F. Let $M$ denote a closed 3-manifold and suppose $S$ is a 2-sphere in $M$ so that $M-S$ is connected. Then there is a 2-sphere $S_{1}$ in $M$ so that $M-S_{1}$ consists of two components $M_{0}$ and $\bar{M}$ where $\pi_{1}\left(M_{0}\right) \approx Z$.

The inductive hypothesis applies to the 3-manifold obtained from $\bar{M}$ by sewing a 3-cell $B_{1}$ onto $S_{1}$ along $\mathrm{Bd} B_{1}$.

Case $2 . \pi_{1}(M)$ is freely reduced.

We proceed as in Case 1 only now we apply Theorem 3.3.

\section{BIBLIOGRAPHY}

1. D. B. A. Epstein, Free products with amalgamation and 3-manifolds, Proc. Amer. Math. Soc. 12 (1961), 669-670. 
2. H. Kneser, Geschlossene Flächen in dreidimensionalen Mannigfaltigkeiten, Jber. Deutsch. Math.-Verein. 38 (1929), 248-260.

3. A. G. Kurosh, The theory of groups. I, II, 2nd ed., Chelsea, New York, 1960.

4. C. D. Papakyriakopoulos, On Dehn's lemma and the asphericity of knots, Ann. of Math. (2) 66 (1957), 1-26.

5. - Some problems on 3-dimensional manifolds, Bull. Amer. Math. Soc. 64 (1958), 317-335.

6. J. Stallings, Grushko's theorem. II. Kneser's conjecture, Notices Amer. Math. Soc. 6 (1959), Abstract 559-165, pp. 531-532.

7. - A topological proof of Grushko's theorem on free products, Math. Z. 90 (1965), 1-8.

8. J. H. C. Whitehead, On finite cocycles and the sphere theorem, Colloq. Math. 6 (1958), 271-281.

9. E. C. Zeeman, Seminar on combinatorial topology, Mimeographed notes, Inst. Hautes Etudes Sci. Publ. Math., 1963.

University of Michigan, Ann Arbor, Michigan 48104 